\title{
Auf dem Weg zur ICD-11
}

\section{On the way to ICD-11}

\author{
Wolfgang Gaebel
}

Klinik und Poliklinik für Psychiatrie und Psychotherapie, LVR-Klinikum Düsseldorf/Klinken der Heinrich-Heine-Universität Düsseldorf

Für die 1992 eingeführte, vorerst weiter gültige 10. Revision (ICD-10) der International Classification of Diseases and Related Health Problems der Weltgesundheitsorganisation (WHO) war bereits 2007 von der World Health Assembly (WHA), der Versammlung der 194 Mitgliedsstaaten der WHO, eine Revision der ICD-10 wegen inhaltlicher und technischer Unzulänglichkeiten in Auftrag gegeben worden (Jakob, 2018). Für die Leitung der Revision des Kapitels F der psychischen Störungen war das WHO Department of Mental Health and Substance Use zuständig; zugleich war eine International Advisory Group for the Revision of ICD-10 Mental and Behavioural Disorders (2011) gegründet worden, um den konzeptuellen, wissenschaftlichen und methodischen Rahmen der Revision für diese Störungsgruppe abzustecken. Entgegen der ursprünglichen Planung konnte allerdings erst 2018, 5 Jahre später als geplant, die 11. Revision (ICD-11) von der WHO offiziell vorgestellt und im Mai 2019 von der WHA ratifiziert werden. Zum 1. Januar 2022 hat die WHO den Beginn des weltweiten Umstiegs von der ICD-10 auf auf ICD-11 zunächst für die Berichterstattung anhand der neuen Mortality and Morbidity Statistics (MMS) in Aussicht gestellt.

Die Implementierung der ICD-11 in den Mitgliedsländern der WHO ist seitdem auf unterschiedlichem Entwicklungsstand. Für Deutschland wird von den einschlägigen Gremien (z.B. Bundesinstitut für Arzneimittel und Medizinprodukte [BfArM]) noch mit einer mehrjährigen Vorbereitungszeit gerechnet. Nicht zuletzt spielt dabei auch die bisher aus verschiedenen Gründen noch nicht vorliegende offizielle deutsche Übersetzung eine Rolle, abgesehen von den Anforderungen der involvierten Gesundheitsorganisationen einschließlich Bundesministerium für Gesundheit (BMG), die in der vom Kuratorium für Fragen der Klassifikation im Gesundheitswesen (KKG) und dem BfArM verantwortlich organisierten nationalen Arbeitsgruppe ICD-11 über die Vielzahl erforderlicher Umsetzungsmaßnahmen und deren Vereinbarkeit (z.B. Interoperabilität der IT von ICD-11 und Gesundheitssystem, Rückwirkungen auf das Entgeltsystem) beraten.
Hierzu zählt vor allem die Frage der Überleitung von ICD10-GM (German Modification) zu ICD-11-GM mit einer Reihe von Spezialproblemen, wie Folgen von Mapping-Inkonsistenzen zwischen ICD-10 und ICD-11 aufgrund struktureller diagnostischer Veränderungen, resultierende diagnostische Crosswalks und mögliche Diskontinuitäten der Caseness zwischen den beiden Versionen, etwaige Prävalenzverschiebungen mit klinisch-therapeutischen Konsequenzen und andere Übergangsphänomene. Natürlich geht es bei dieser Problematik nicht nur oder primär um die psychomedizinischen Fachdisziplinen. Für das Kapitel der psychischen und angrenzenden Störungen der ICD-11 hat die WHO eine International WHO Advisory Group on Training and Implementation zum Erfahrungsaustausch einberufen. Störungsübergreifende Tools der WHO zur systematischen Organisation des Übergangs zwischen den Versionen und der Implementierung von ICD-11 sind im Internet abrufbar.

Als Klassifikation für die gesamte Medizin verfügt die ICD-11 über insgesamt 28 Kapitel, die neben den Störungskapiteln i.e.S. eine Reihe von Kapiteln für störungsübergreifende Zusatzcodes beinhalten. Für die psychomedizinischen Disziplinen ergaben sich eigene Problemstellungen in der Entwicklung der ICD-11, bei deren Beratung die eingangs genannte Kommission unter Leitung von S. Hyman (US National Institute of Mental Health [NIMH]) eine Rolle spielte. Die entwickelte Metastruktur der ICD-11 MMS (https://icd.who.int/browse11/1-m/en) des neuen Kapitels 06 Mental, behavioural or neurodevelopmental disorders (MBND) folgte im Gegensatz zur ICD-10 und in gewisser Abstimmung mit der Revision von DSM-IV (Diagnostic and Statistical Manual of Mental Disorders; First et al., 2021) der Entwicklungsperspektive über die Lebensspanne - in stärker gegliederter Struktur dank alphanumerischer Kodiermöglichkeiten - mit den neurodevelopmental disorders am Beginn und den neurocognitive disorders am Ende des Kapitels. Bisher im F-Kapitel der ICD-10 verortete Störungen finden sich nun in jeweils eigenen Kapiteln, wie Sleep-wake disorders [Kapitel 07] oder Conditions related 
to sexual health [Kapitel 17]. Neurologische bzw. im Überlappungsbereich zwischen Neurologie und Psychiatrie liegende Störungen finden sich im Kapitel 08 Diseases (!) of the nervous system. Analog zur Kreuz-Stern Verschlüsselung der Kapitel F00-F09 der ICD-10 werden klinische Manifestationen neurokognitiver Störungen in der ICD-11 in Kapitel 06 kodiert und obligatorisch mit einem Code zur Ätiopathogenese in Kapitel 08 postkoordinativ kombiniert. Es dürfte hier in den kommenden Jahren zwischen den Fächern zu weiteren Änderungsvorschlägen, Diskussionen und bestenfalls Einvernehmlichkeiten unter Nutzung des vorhandenen Vorschlagstools kommen (Gaebel, Reed \& Jakob, 2019), solange Manifestation und Ätiopathogenese neuropsychiatrischer Störungsbilder auf unterschiedliche Fachkapitel verteilt bleiben. Der im Internet verfügbare Reference Guide der WHO gibt einen sehr guten Überblick über alles Wissenswerte zur ICD-11, insbesondere auch zu Struktur und Taxonomie der ICD als Klassifikationssystem.

Die Arbeitsgruppen für die Weiterentwicklung der einzelnen Störungskapitel waren themenspezifisch von internationalen Expertinnen und Experten besetzt und geleitet, in enger Abstimmung mit dem WHO-Department (repräsentiert durch G.M. Reed, Senior Project Officer). Ergebnis der mehrjährigen Arbeit waren die Draftversionen der CDDG (Clinical Description and Diagnostic Guidelines), strukturiert nach einer „Content Form“ mit jeweils essenziellen diagnostischen Merkmalen (möglichst flexible Anwendung ohne operationale Pseudopräzision oder Polythetik), Abgrenzung gegenüber anderen psychischen Störungen (Differenzialdiagnose) oder Normvarianten sowie Berücksichtigung kultur- und geschlechterspezifischer Reaktionen etc. (First, Reed, Hyman \& Saxena, 2015; Reed et al., 2019). Die CDDG wurden auf der GCPN (Global Clinical Practice Network)-Internetplattform der WHO kontinuierlich weiterentwickelt und abgelegt (https:/gcp.network), einem Netzwerk klinischer Expertinnen und Experten vorwiegend aus Psychiatrie und Psychologie, mit derzeit ca. 16000 Mitgliedern (wobei Deutschland Platz 4 der Mitgliederanzahl belegt), wo die CDDG nach persönlicher Registrierung abgerufen werden können und von wo aus sie derzeit in die Foundation der ICD-11 überführt werden als Grundlage für autorisierte Übersetzungen mit dem WHO Translation Tool.

Insgesamt umfasst das Kapitel MBND 21 Störungskapitel im Vergleich zu zehn Störungskapiteln des Kapitels F der ICD-10. Innerhalb und zwischen den einzelnen Kapiteln gibt es zum Teil erhebliche Veränderungen; 15 Subkapitel wurden gänzlich neu eingefügt (Gaebel, Stricker \& Kerst, 2020; Reed et al., 2019), wobei einzelne Subkapitel, wie z.B. Prolonged grief disorder, Gaming disorder oder Compulsive sexual behaviour disorder nach Bekanntwerden erhebliche Diskussion in den Medien bezüglich einer etwaigen „Psychiatrisierung“ hervorgerufen haben.
In der Entwicklung der CDDG für die Kapitel der MBND der ICD-11 standen als Leitkonzepte wissenschaftliche Validität, diagnostische Konsistenz (,Reliability“), „Utility“ und „Global Applicability“ im Vordergrund. Die ICD-11 ist auch weiterhin ein überwiegend kategorial strukturiertes Klassifikationssystem, wenn man von den Persönlichkeitsstörungen absieht, wobei ein erheblicher Zuwachs an dimensional konfigurierten „Specifiers“ oder „Qualifiers“ zu verzeichnen ist, die im Bereich von Symptom-, Verlaufsund Schweregradindikatoren angesiedelt sind und zusätzlich zu teilweise präkoordinierten Kategorien (siehe z.B. Schizophrenia or other primary psychotic disorders) postkoordinativ hinzugefügt werden können. Das entsprechende „Coding Tool“ für ICD-11 ermöglicht eine automatisierte Kombination $\mathrm{zu}$ entsprechend extendierten Codestrings i.S. von Komplexcodes, die eine stärker personalisierte Diagnostik und Therapieindikation erlauben. $\mathrm{Ob}$ in Zukunft stärker dimensional orientierte diagnostische Konzeptentwicklungen wie z.B. HiTOP (Hierarchical Taxonomy of Psychopathology) eine praktische klinische Bedeutung erlangen werden, mag dahinstehen (Gaebel et al., 2020). Die zu Beginn der Revision für DSM-5 wie ICD11 intendierte stärkere Berücksichtigung von Biomarkern für die Diagnostik musste seinerzeit mangels hinreichender Evidenz und praktischer Relevanz für beide Systeme zunächst aufgegeben werden, dürfte aber bei künftig in engeren Zeiträumen stattfindenden Revisionen weiter auf der Agenda stehen. Zunächst ist das Ziel erreicht worden, diagnostische Reliabilität, „Utility“ und „Applicability“ für die Mehrzahl von Diagnosen des Kapitels MBND der ICD-11 im Vergleich zu Diagnosen des Kapitels F der ICD10 in internetbasierten (mit Fallvignetten) und klinischen Feldstudien an internationalen Zentren zu verbessern oder als mindestens vergleichbar gut zu sichern (Reed, Keeley, et al., 2018; Reed, Sharan, et al., 2018).

Wenn auch weder für DSM-5 noch für ICD-11 ein „Paradigmenwechsel“ gegenüber der jeweiligen Vorversion zu verzeichnen ist, liegt doch mit dem Kapitel MBND der ICD11 eine Revision der ICD vor, die eine Reihe von wesentlichen Innovationen, aber auch Herausforderungen an das jeweilige nationale Gesundheitssystem mit sich bringt. Mit den Möglichkeiten einer regelmäßigen „Maintenance“ und von Updates sowie der Einbringung von Kommentaren und Änderungsvorschlägen auf der „Proposal“-Plattform, die unter der Kontrolle von „Governance“-Strukturen der WHO mit beratenden Kommissionen wie MSAC (Medical Scientific Advisory Committee) und CSAC (Classification and Statistics Advisory Committee) stehen, sind Anpassungsmöglichkeiten an den Fortschritt in Wissenschaft und Praxis weiter gegeben. Damit wird die ICD-11 auch dem Bedarf einer globalen Reform von „Mental health and care“ im Sinne einer stärker personalisierten Versorgung besser gerecht. Im Hinblick auf Übergang und Implementierung, 
zu deren Unterstützung die WHO eigens einen „ICD-11 Implementation or Transition Guide" (im Internet verfügbar) zur Verfügung hält, wird deutlich, was ein solcher Schritt national an Aufwand erfordert. Nicht zuletzt „Education“ und „Training“ der „Mental health workforce“ im Bereich von MBND sind hier flächendeckend zu organisieren, wobei sich der Bedarf bereits jetzt bei entsprechenden virtuellen Kursangeboten einiger Fachgesellschaften, wie Royal College of Psychiatry, European Psychiatric Association (EPA) oder World Psychiatric Association (WPA), in einer zunehmend starken Nachfrage niederschlägt.

Die im Folgenden in diesem Themenheft der Zeitschrift für Kinder- und Jugendpsychiatrie versammelten Beiträge geben einen hoch informativen wie kritischen Einblick in fachspezifisch ausgewählte diagnostische Kategorien der ICD-11 (Arnaud \& Thomasius, 2021; Freitag, 2021; GörtzDorten \& Döpfner, 2021; Gradl-Dietsch, Herpertz-Dahlmann, Degenhardt \& Hebebrand, 2021; Herpertz-Dahlmann, 2021; Schmeck \& Birkhölzer, 2021; Schultze-Lutter, Meisenzahl \& Michel, 2021; Vloet \& Romanos, 2021; von Gontard, 2021): Was ist auf dem Weg von der ICD-10 zur ICD-11 aus der Fachperspektive neu und ggf. kritisch zu bewerten? Wie wird der an der Lebensspanne orientierte Zugang zu den diagnostischen Kategorien im Fach unter dem Aspekt einer Transitionspsychiatrie beurteilt? Welche Ausblicke ergeben sich in klinisch-praktischer und wissenschaftlicher Hinsicht mit welchem weiteren Forschungsbedarf? Und wie fällt der Vergleich mit DSM-5 aus? Diesen Fragen stellen sich die einzelnen Beiträge mit Kennerschaft und eindeutigem Kenntnisgewinn für die Leserschaft. Nicht alle Störungsbilder werden schon in diesem Heft behandelt. In loser Folge werden auch künftig noch Beiträge zu weiteren Diagnosen folgen.

\section{Literatur}

Arnaud, N. \& Thomasius, R. (2021). Störungen durch Substanzgebrauch und abhängige Verhaltensweisen in der ICD-11. Zeitschrift für Kinder- und Jugendpsychiatrie und Psychotherapie, 49, 487-493. doi: 10.1024/1422-4917/a000748

First, M.B., Gaebel, W., Maj, M., Stein, D.J., Kogan, C.S., Saunders, J.B. et al. (2021). An organization- and category-level comparison of diagnostic requirements for mental disorders in ICD-11 and DSM-5. World Psychiatry, 20, 34-51. doi:10.1002/wps.20825

First, M. B., Reed, G. M., Hyman, S. E. \& Saxena, S. (2015). The development of the ICD-11 Clinical Descriptions and Diagnostic Guidelines for Mental and Behavioural Disorders. World Psychiatry, 14, 82-90. doi: 10.10012/wps.20189

Freitag, C.M. (2021). Von den tiefgreifenden Entwicklungsstörungen in ICD-10 zur Autismus-Spektrum-Störung in ICD-11. Zeitschrift für Kinder- und Jugendpsychiatrie und Psychotherapie, 49, 437-441. doi: 10.1024/1422-4917/a000774

Gaebel, W., Reed, G.M. \& Jakob, R. (2019). Neurocognitive disorders in ICD-11: A new proposal and its outcome. World Psychiatry, 8, 232-233. doi:10.1002/wps.20634
Gaebel, W., Stricker, J. \& Kerst, A. (2020). Changes from ICD-10 to ICD-11 and future directions in psychiatric classification. Dialogues in Clinical Neuroscience, 22, 7-15. doi: 10.31887/DCNS. 2020.22.1/wgaebel

Görtz-Dorten, A. \& Döpfner, M. (2021). Störungen mit oppositionellem und trotzigem Verhalten und dissoziale Verhaltensstörungen. Zeitschrift für Kinder- und Jugendpsychiatrie und Psychotherapie, 49, 494-498. doi: 10.1024/1422-4917/a000764

Gradl-Dietsch, G., Herpertz-Dahlmann, B., Degenhardt, F. \& Hebebrand, J. (2021). ICD-11-Sonderserie: Fütter- und Essstörungen in der ICD-11. Zeitschrift für Kinder- und Jugendpsychiatrie und Psychotherapie, 49, 443-452. doi:10.1024/1422-4917/a000772.

Herpertz-Dahlmann, B. (2021). Klassifikation der dissoziativen Störungen und der Störung der körperlichen Belastung - ein Vergleich zwischen ICD-10 und ICD-11. Zeitschrift für Kinderund Jugendpsychiatrie und Psychotherapie, 49, 417-420. doi: 10.1024/1422-4917/a000745

International Advisory Group for the Revision of ICD-10 Mental and Behavioural Disorders. (2011). A conceptual framework for the revision of the ICD-10 classification of mental and behavioural disorders. World Psychiatry, 10, 86-92.

Jakob, R. (2018). ICD-11 - Anpassung der ICD-11 an das 21. Jahrhundert. Bundesgesundheitsblatt - Gesundheitsforschung - Gesundheitsschutz, 61, 771-777. doi.org/10.1007/s00103-018-2755-6

Reed, G. M., First, M.B., Kogan, C.S., Hyman, S. E., Gureje, O., Gaebel, W. et al. (2019). Innovations and changes in the ICD-11 classification of mental, behavioural and neurodevelopmental disorders. World Psychiatry, 18, 3-19. doi:10.1002/wps.20611

Reed, G. M., Keeley, J. W., Rebello, T.J., First, M. B., Gureje, O., AyusoMateos, J. L. et al. (2018). Clinical utility of ICD-11 guidelines for high-burden mental disorders: Results from mental health settings in 13 countries. World Psychiatry, 7, 306-315. doi:10.1002/ wps. 20581

Reed, G.M., Sharan, P., Rebello, T.J., Keeley, J.W., Medina-Mora, M. E., Gureje, O. et al. (2018). The ICD-11 developmental field study of reliability of diagnoses of high-burden mental disorders: Results among adult patients in mental health settings of 13 countries. World Psychiatry, 7, 174-186. doi:10.1002/wps.20524

Schmeck, K. \& Birkhölzer, M. (2021). Die Konzeption von Persönlichkeitsstörungen in der ICD-11. Zeitschrift für Kinder- und Jugendpsychiatrie und Psychotherapie, 49, 480-485. doi: 10.1024/1422-4917/a000747

Schultze-Lutter, F., Meisenzahl, E. \& Michel, C. (2021). Psychotische Störungen in der ICD-11: Die Revisionen. Zeitschrift für Kinder- und Jugendpsychiatrie und Psychotherapie, 49, 453462. doi: 10.1024/1422-4917/a000777

Vloet, T.D. \& Romanos, M. (2021). Angststörungen - von der ICD-10 zur ICD-11. Zeitschrift für Kinder- und Jugendpsychiatrie und Psychotherapie, 49, 429-435. doi: 10.1024/1422-4917/a000768

von Gontard, A. (2021). Ausscheidungsstörungen - Klassifikation und Definition nach ICD-11. Zeitschrift für Kinder- und Jugendpsychiatrie und Psychotherapie, 49, 421-428. doi:10.1024/14224917/a000751

\section{Univ.-Prof. Dr. med. Wolfgang Gaebel}

European Scientific Association on Schizophrenia (ESAS) WHO Collaborating Centre DEU-131

Klinik und Poliklinik für Psychiatrie und Psychotherapie LVR-Klinikum Düsseldorf

Kliniken der Heinrich-Heine-Universität Düsseldorf Bergische Landstr. 2

40629 Düsseldorf

Deutschland

wolfgang.gaebel@uni-duesseldorf.de 